\title{
Biochemical Markers of Bone Turnover as Predictors of Bone Mineral Density Response to Denosumab
} \author{
Mahillo Fernández ${ }^{3}$ and Manuel Díaz Curiel ${ }^{1 *}$ \\ ${ }^{1}$ Department of Internal Medicine, Fundación Jiménez Díaz Hospital, Spain \\ ${ }^{2}$ Department of Internal medicine, Infanta Leonor Hospital, Spain \\ ${ }^{3}$ Epidemiology and Bioestatistics Unit, Fundación Jiménez Díaz, Spain
}

Natalia Bravo Martín ${ }^{1}$, María Jesús Moro Álvarez ${ }^{2}$, Rosa María Arboiro Pinel ${ }^{1}$, Marjorie Andrade ${ }^{1}$, Ignacio

Submission: November 17, 2017; Published: November 28, 2017

*Corresponding author: Manuel Díaz Curiel, Metabolic Bone Diseases Unit, Department of Internal Medicine, Fundación Jiménez Díaz Hospital, Madrid, Spain, Email: MDCuriel@fjd.es

\begin{abstract}
Introduction: In osteoporosis, there is an increase in reabsorption and a decrease in bone formation. This bone turnover could be measured through bone turnover markers (BTM) in order to predict the response in bone mineral density. In this trial, we describe BTM after initiation of Denosumab (DMAB) treatment and its relationship with subsequent long-term responses of BMD (Bone Mineral Density).

Materials and Methods: We observed 104 patients with osteoporosis in treatment with DMAB $60 \mathrm{mg}$, with subcutaneous administration every six months, between 2012 and 2016. The $46.5 \%$ of patients had received previous treatment with bisphosphonates and the rest of them were naïve patients. We determined the values of markers of bone turnover (PINP and CTX) at baseline, then at 6 months and 12 months after starting treatment with DMAB and BMD was measured at baseline, then at 12 months and 24 months, after the initiation of DMAB. Finally, we verified whether there is a correlation between the decrease in bone markers at 6 months and the increase in BMD at one year.

Results and Conclusion: This analysis confirms a relationship between the descent of bone turnover marker and BMD response to therapy with DMAB; in this case between P1NP and CTX at 6 months of initiation of treatment and the increase in BMD in lumbar spine (LS), femoral neck (FN) and total radius (TR) at 1 year, but not statistically significant. We performed a sub analysis excluding the patients who were previously treated with bisphosphonates, finding a correlation between decreased levels of CTX and P1NP at 6 months and the increase in BMD in FN and TF at 1 year, respectively and statistically significant. We also detected a correlation between a decreased of P1NP at 6 months and the increase in BMD in TF at 2 years, statistically significant. We conclude that the use of bisphosphonates plays a role as confusion factor probably explaining why it is not statistically significant the whole analysis of the study. Therefore, future analysis will be needed. There is insufficient evidence to choose bone turnover markers to monitor osteoporosis treatment response in routine clinical practice, although it would be interesting to measure them in order to help us predict the therapeutic response at the level of bone mineral density.
\end{abstract}

Keywords: Osteoporosis; Denosumab; Biochemical markers of bone turnover; BMD; BMT; Clinical trials; Monitoring response

\section{Introduction}

Osteoporosis is a systemic disease characterised by low bone mass and an impaired micro architecture of bone, with the consequent increase in bone weakness and elevated risk of falls and fractures. It constitutes a public health problem worldwide and his prevalence is increasing, with the consequent impact on population: hospitalization, lower quality of life, important cause of morbidity and mortality and increased spending on the health system. An increase in bone remodelling has been observed in patients with osteoporosis. As a result, products of osteoclast and osteoblast activity could be modified and measured as biochemical markers in serum in order to predict the response in bone mineral density (BMD) [1].

There are some available osteoporosis treatments; which can be classified in two groups: a) Antiresorptive agents like bisphosphonates (BP), selective estrogen receptor modulators (SERM) and denosumab (DMAB) and,

b) Bone-forming agents such as teriparatide and new agents such as Anti-sclerostin antibodies.

In addition, Strontium ranelate (SR), with a dual mechanism of action [2].

Denosumab is a human monoclonal antibody against the receptor activator of nuclear factor kappa-B ligand (RANKL) used for increasing bone mass in patients with osteoporosis and high risk for fracture. In previous studies, it has been shown that it is a potent antiresorptive agent that increases bone mass significantly at lumbar spine, total hip and one-third 
radius compared with placebo, and reduced the risk of vertebral and nonvertebral fractures in postmenopausal women with osteoporosis [3]. A few studies have evaluated correlations between biochemical markers and the BMD response with the different treatments of osteoporosis [4]. In 2005, Peiqui Chen et al. [1] had carried out a trial to quantify the relationship between biochemical markers and the BMD responses to teriparatide at the lumbar spine and femoral neck, observing an increase in markers 1 month after initiation of therapy. They found a highest correlation between the change in PINP (Carboxy-terminal extension peptide of procollagen type 1) at 3 months and the lumbar spine BMD response at 18 months.

Other Clinical trials demonstrated that determinations of biochemical markers of bone turnover could be used to monitor bisphosphonates treatment and that biochemical markers like NTX (N-telopeptide cross-links of type I collagen) and osteocalcin were significantly correlated with change from baseline at month 24 in spine and hip BMD [5]. Delmas PD et al. [6], observed that treatment with hormone replacement therapy (HRT) induces a rapid decrease in biochemical markers of bone which correlates with a subsequent increase in BMD. They correlated the changes in 3 markers (osteocalcin, ALP and CTX) and BMD in 569 women treated with 17 beta-estradiol, for 2 years, objectifying that resorption marker at 3 and 6 months of treatment were predictive of BMD response at 2 years [6].

A small study with a total of 13 female patients treated with SR after 4 months and previously treated with BP show that SR has a short-term predominantly stimulating effect on bone markers (Osteocalcin and CTX), which is associated with a increase in lumbar spine BMD at 2.5 years of treatment with SR [7]. The aim of this study was to describe bone turnover marker (ALP, P1NP and CTX) baseline and at 6 and 12 months after initiation of Denosumab treatment and its relationship with subsequent long-term responses of BMD at 12 and 24 months in LS, TF, FN and radius, to considering these markers as shortterm predictors of changes in bone mass.

\section{Material and Methods}

\section{Study population}

The present study, which was carried out from March 2012 to June 2016, included 104 patients of the metabolic bone disease unit in our centre, including 101 postmenopausal women and 3 men aged 41 to 89 (mean of 72 years old), who were in treatment with DMAB with subcutaneous administration every six months. The $46.5 \%$ of patients had received previous treatment with BP, $14.6 \%$ with teriparatide and $19.5 \%$ did not receive any previous treatment.

\section{Bone Turnover Markers Assessment}

From the fasting blood sample, we determined the values of some analytical variables like levels of calcium, creatinine, paratohormone, vitamin $\mathrm{D}$, alkaline phosphatase and we assessed markers of bone remodelling as P1NP and a resorption marker as CTX, using electrochemiluminescence and radioimmunoassay, respectively. These determinations were performed at baseline and at 6 months and 12 months after starting treatment with DMAB. We described the changes in the markers at 6 and 12 months with respect to the baseline, showing the mean and standard deviation, as well as the p-value obtained by the Student's T test.

\section{Bone Mineral Density Assesment (BMD)}

BMD of lumbar spine (LS), total femur (TF), femoral neck (FN) and total radius (TR) in some patients, was measured using DXA (Hologic c.v. In vivo 1,2\%) at baseline at 12 months and 24 months, after the initiation of DMAB, describing the changes at 6 and 12 months with respect to the baseline, obtained by the Student's T test.

\section{Correlations between Biochemical Markers of Bone Turnover and BMD Response}

Finally, we verified whether there is a correlation, through the Spearman coefficient, between the decrease in bone markers at 6 months and the increase in BMD at one year.

\section{Results}

Table 1: Same analysis as in the previous point (Table 2), observing as a result a significant increase of BMD at 1 year and at 2 years of treatment with DMAB at LS and FN levels, without being significant in TF and TR.

\begin{tabular}{|c|c|c|c|c|}
\hline Variable & Changes at 6 months & & Changes at 12 months & \\
\hline Creatinine & $0.019 \pm 0.141$ & 0.48 & $-0.034 \pm 0.138$ & 0.196 \\
\hline Calcium & $-1.639 \pm 8.461$ & 0.289 & $-1.629 \pm 8.412$ & 0.290 \\
\hline Vitamin D & $1.407 \pm 19.609$ & 0.657 & $-0.429 \pm 17.455$ & 0.879 \\
\hline PTH & $1.871 \pm 19.179$ & 0.573 & $4.988 \pm 21.431$ & $<0.001$ \\
\hline ALP & $-15.07 \pm 16.67$ & $<0.001$ & $-14.45 \pm 16.49$ & $<0.001$ \\
\hline CTX & $-0.176 \pm 0.211$ & $<0.001$ & $-23.28 \pm 20.91$ & $<0.001$ \\
\hline P1NP & $-19.92 \pm 23.79$ & $<0.001$ & & \\
\hline
\end{tabular}




\section{Orthopedics and Rheumatology Open Access Journal}

Table 2: There is no statistically significant correlation between the decrease in CTX and P1NP at 6 months and the increase in BMD at LS, FN and TR.

\begin{tabular}{|c|c|c|c|c|}
\hline Variable & Changes at 12 months & & Changes at 24 months & $<0.001$ \\
\hline BMD LS & $0.050 \pm 0.054$ & $<0.001$ & $0.066 \pm 0.056$ & $<0.001$ \\
\hline T-Score LS & $0.437 \pm 0.498$ & $<0.001$ & $0.582 \pm 0.469$ & $<0.001$ \\
\hline BMD FN & $0.015 \pm 0.029$ & 0.003 & $0.023 \pm 0.032$ & 0.076 \\
\hline T-Score FN & $0.159 \pm 0.719$ & 0.186 & $0.101 \pm 0.518$ & 0.25 \\
\hline BMD TF & $0.096 \pm 0.516$ & 0.272 & $0.014 \pm 0.656$ & 0.898 \\
\hline T-Score TF & $0.020 \pm 0.615$ & 0.849 & $0.009 \pm 0.019$ & 0.039 \\
\hline BMD TR & $0.007 \pm 0.018$ & 0.092 & $0.333 \pm 0.720$ & 0.047 \\
\hline T-Score TR & $0.110 \pm 0.308$ & 0.119 & & \\
\hline
\end{tabular}

Table 3: Correlation between markers at 6 months and BMD at 1 year in all patients.

\begin{tabular}{|c|c|c|c|}
\hline Markers & DXA & Correlation & P \\
\hline \multirow{4}{*}{ CTX } & BMD LS & -0.18 & -0.18 \\
\cline { 2 - 4 } & BMD FN & -0.18 & -0.176 \\
\cline { 2 - 4 } & BMD TF & 0.08 & 0.081 \\
\cline { 2 - 4 } & BMD TR & -0.09 & -0.094 \\
\hline \multirow{4}{*}{ P1NP } & BMD LS & -0.01 & -0.009 \\
\cline { 2 - 4 } & BMD FN & -0.07 & -0.071 \\
\cline { 2 - 4 } & BMD TF & 0.06 & 0.06 \\
\cline { 2 - 4 } & BMD TR & -0.1 & -0.096 \\
\hline
\end{tabular}

Table 4: Correlation between BTM at 6 months and BMD at 12 months in naive patients.

\begin{tabular}{|c|c|c|c|c|}
\hline Markers & DXA & Correlation & & $\mathbf{p}$ \\
\hline \multirow{4}{*}{ CTX } & BMD LS & -0.16 & $(-0.56,0.29)$ & 0.478 \\
\cline { 2 - 5 } & BMD FN & -0.43 & $(-0.73,0.00)$ & 0.051 \\
\cline { 2 - 5 } & BMD TF & -0.05 & $(-0.49,0.42)$ & 0.847 \\
\cline { 2 - 5 } & BMD TR & -0.23 & $(-0.68,0.34)$ & 0.423 \\
\hline \multirow{4}{*}{ P1NP } & BMD LS & 0.23 & $(-0.23,0.60)$ & 0.319 \\
\cline { 2 - 5 } & BMD FN & -0.31 & $(-0.66,0.14)$ & 0.165 \\
\cline { 2 - 5 } & BMD TF & -0.53 & $(-0.79,-0.1)$ & 0.02 \\
\cline { 2 - 5 } & BMD TR & -0.28 & $(-0.72,0.32)$ & 0.355 \\
\hline
\end{tabular}

Table 5: Correlation between BTM at 6 months and BMD at 24 months in naive patients.

\begin{tabular}{|c|c|c|c|c|}
\hline Markers & DXA & Correlation & & $\mathbf{p}$ \\
\hline \multirow{4}{*}{ CTX } & BMD LS & -0.43 & $(-0.79,0.16)$ & 0.143 \\
\cline { 2 - 5 } & BMD FN & 0.06 & $(-0.51,0.59)$ & 0.843 \\
\cline { 2 - 5 } & BMD TF & 0.09 & $(-0.49,0.61)$ & 0.782 \\
\cline { 2 - 5 } & BMD TR & 0.07 & $(-0.59,0.67)$ & 0.851 \\
\hline \multirow{4}{*}{ P1NP } & BMD LS & 0.2 & $(-0.40,0.68)$ & 0.517 \\
\cline { 2 - 5 } & BMD FN & -0.08 & $(-0.61,0.49)$ & 0.791 \\
\cline { 2 - 5 } & BMD TF & -0.58 & $(-0.86,-0.04)$ & 0.039 \\
\cline { 2 - 5 } & BMD TR & 0.15 & $(-0.57,0.74)$ & 0.709 \\
\hline
\end{tabular}

Data was available for 104 patients treated with DMAB. Table 1 describes the baseline values and changes at 6 and 12 months of the analytical values and markers of bone remodelling. We found statistical significance for changes in ALP, CTX and P1NP (Tables $2 \& 3$ ). In these three variables we found that there was a reduction, although changes at 6 and 12 months were very similar. We performed a sub analysis excluding the patients who were previously treated with bisphosphonates, finding a correlation between decreased levels of CTX and P1NP at 6 months and the increase in BMD in FN and TF at 1 year, respectively and statistically significant (Table 4). We also detected a correlation between a decreased of P1NP at 6 months and the increase in BMD in TF at 2 years, statistically significant (Table 5).

\section{Discussion}

Specific markers to bone formation include bone-specific alkaline phosphatase (ALP), N-terminal propeptide of type 1 procollagen (P1NP) and osteocalcin; Bone resorption's markers include N-telopeptide of type 1 collagen (NTX) and C-terminal telopeptide of type 1 collagen (CTX). In terms of mechanisms of action, therapy with antiresorptive agents reduce bone turnover, which causes the stabilisation or an increase in BMD. Treatment with a bone-forming agent like teriparatide, in contrast to antiresorptive agents, increases bone remodelling, promoting an increase in the production of bone markers [2]. Actually, there are no guidelines for monitoring patients receiving treatment for osteoporosis, although usually they are monitored by dualenergy X-ray absorptiometry (DXA) with a minimum of 12-24 months between each of them. The use of bone remodelling markers has advantages over DXA: a cheaper procedure, less invasive and also can detect changes in bone turnover earlier. However, the use of such markers has other disadvantages; especially the variability between patients so they are not used routinely [4].

\section{Conclusion}

Bone turnover markers can provide information on the response to osteoporosis therapy rapidly, because of the reflex of the situation of bone activity. Actually, P1NP and CTX are the two bone markers most used in the clinical practice. Many studies have shown that different drugs for osteoporosis produce changes in these markers and increase bone mass. However, few studies correlate these markers with the situation of bone mass. This analysis confirms a relationship between baseline bone turnover marker and BMD response to therapy with Denosumab, 
in this case between the descent of P1NP y CTX at 6 months of initiation of treatment and the increase in BMD in femoral neck and total femur, respectively, statistically significant and also between the descent of P1NP at 6 months and the increase in BMD in total femur at 2 years, in naïve patients.

We conclude that the use of bisphosphonates plays a role as confusion factor probably explaining why it is not statistically significant the whole analysis of the study. Therefore, future analysis will be needed. There isn't sufficient evidence to choose bone turnover markers to monitor osteoporosis treatment response in routine clinical practice, although it would be interesting to measure them in order to help us predicting the therapeutic response at the level of bone mineral density before BMD changes can be evaluated [8-10].

\section{References}

1. Chen P, Satterwhite JH, Licata AA, Lewiecki EM, Sipos AA, et al. (2005) Early changes in biochemical markers of bone formation predict BMD response to teriparatide in postmenopausal women with osteoporosis. J Bone Miner Res 20: 962-970.

2. Tabatabaei-Malazy O, Salari P, Khashayar P, Larijani B (2017) New horizons in treatment of osteoporosis. DARU 25(1): 2.

3. Sánchez A, Brun LR, Salerni H, Costanzo PR, González D, et al. (2016) Effect of Denosumab of bone mineral density and markers of bone turnover among postemenopausal women with osteoporosis. J Osteoporosis 2016: 8738959.
4. Burch J, Rice S, Yang H, Neilson A, Stirk L, et al. (2014) Systematic review of the use of bone turnover markers for monitoring the response to osteoporosis treatment: the secondary prevention of fractures, and primary prevention of fractures in high-risk groups. Health Technology Assessment 18(11): 1-180.

5. Ravn P, Hosking D, Thompson D, Cizza G, Wasnich RD, et al. (1999) Monitoring of alendronate treatment and prediction of effect on bone mass by biochemical markers in the early postmenopausal intervention cohort study. J Clin Endocrinol Metab 84(7): 2363-2368.

6. Delmas PD, Hardy P, Garnero P, Dain M (2000) Monitoring individual response to hormone replacement therapy with bone markers. Bone 26(6): 553-560.

7. Helisane Lima, Juliana Maia, Francisco Bandeira (2014) Trajectories of bone remodeling markers and bone mineral density during treatment with strontium ranelate in postmenopausal women previously treated with bysphosphonates. Clinical Medicine Insights: Endocrinology and Diabetes 7: 7-14.

8. Patrick Garnero (2017) The utility of biomarkers in osteoporosis management. Mol Diagn Ther 21(4): 401-418.

9. Naylor K, Eastell R (2012) Bone turnover markers: use in osteoporosis. Nat Rev Rheumatol 8(7): 379-389.

10. Bergmann P, Body JJ, Boonen S, Boutsen Y, Devogelaer JP, et al. (2009) Evidence-based guidelines for the use of biochemical markers of bone turnover in the selection and monitoring of bisphosphonate treatment in osteoporosis: a consensus document of the Belgian Bone Club. Int Clin Pract 63(1): 19-26.

\section{Your next submission with Juniper Publishers will reach you the below assets}

- Quality Editorial service

- Swift Peer Review

- Reprints availability

- E-prints Service

- Manuscript Podcast for convenient understanding

- Global attainment for your research

- Manuscript accessibility in different formats

( Pdf, E-pub, Full Text, Audio)

- Unceasing customer service

Track the below URL for one-step submission https://juniperpublishers.com/online-submission.php 